PROCEEDINGS OF THE

AMERICAN MATHEMATICAL SOCIETY

Volume 130, Number 1, Pages 213-216

S 0002-9939(01)06045-2

Article electronically published on April 25, 2001

\title{
INDEPENDENCE AND ATOMS
}

\author{
GÁBOR J. SZÉKELY AND TAMÁS F. MÓRI \\ (Communicated by Claudia M. Neuhauser)
}

\begin{abstract}
We prove that if the range of a probability measure $P$ contains an interval $[0, \varepsilon], \varepsilon>0$, then there are infinitely many (nontrivial) independent events in this probability space.
\end{abstract}

\section{INTRODUCTION AND RESULTS}

The notion of independence is fundamental in probability theory. Recently it has been proved (3) that two probability measures $P$ and $Q$ on a $\sigma$-field $\mathcal{A}$, where $P$ or $Q$ is atomless, coincide if and only if $P(A B)=P(A) P(B)$ is equivalent to $Q(A B)=Q(A) Q(B), A, B \in \mathcal{A}$.

Recall that an event $A \in \mathcal{A}$ is an atom if $P(A)>0$, and $B \subset A, P(B)<P(A)$ imply $P(B)=0$. Purely atomic means that there is a sequence of atoms such that the sum of their probabilities is 1 .

If the probability measure is purely atomic, then it is certainly possible that there are no nontrivial (= not empty and not the whole space) independent events at all. The following example is a sufficient condition for that. If the probability space is purely atomic, then without loss of generality we can suppose that the underlying probability space is $\left\{\mathbb{N}_{0}, \mathcal{A}, P\right\}$ where $\mathbb{N}_{0}=\{0,1,2, \ldots\}, \mathcal{A}$ is the set of all subsets of $\mathbb{N}_{0}$, and $P$ is defined by a non-increasing sequence $a_{n}=P(n), n \in \mathbb{N}_{0}$.

Example 1.1. If $a_{n+1} \leq a_{n}^{2}$ holds for all $n \in \mathbb{N}_{0}$, then there are no (nontrivial) independent events in this probability space.

For $n>1$, our condition implies that the tail probability

$$
t_{n}=a_{n}+a_{n+1}+\cdots<a_{n} /\left(1-a_{n}\right)<a_{n} / a_{n-1} \leq a_{n-1} \leq a_{n-2}^{2} .
$$

Now, if $A$ and $B$ are two arbitrary nonempty events and the smallest element of $A$, $B$, and $A B$ are $k, m$, and $n$, respectively, then we can suppose $0<k \leq m \leq n$. (If $A$ or $B$ contains 0 , then their complements do not, and the complements are also independent, so we can then switch from $A$ or $B$ to their complements.)

If $m=n$, then

$$
P(A) P(B) \leq t_{k} t_{m}<\left(1-a_{0}\right) a_{m} /\left(1-a_{m}\right)<a_{m} \leq P(A B) .
$$

If $m<n$, then

$$
P(A B) \leq t_{n}=a_{n}+a_{n+1}+t_{n+2}<a_{n-1}^{2}+2 a_{n}^{2}<\left(a_{n-1}+a_{n}\right)^{2}<P(A) P(B) .
$$

Received by the editors July 15, 1999 and, in revised form, May 24, 2000.

2000 Mathematics Subject Classification. Primary 60A10.

Key words and phrases. Independence, atom, purely atomic probability measure, BorelCantelli lemma, range of a probability measure. 
Proposition 1.1. If the probability space contains at least one atom and infinitely many independent events with probabilities $p_{1}, p_{2}, p_{3}, \ldots$, then

$$
\sum_{n=1}^{\infty} \min \left\{p_{n}, 1-p_{n}\right\}<\infty
$$

Conversely, if this sum is finite, then there exist a purely atomic probability space and an infinite sequence of independent events in it with probabilities $p_{1}, p_{2}, p_{3}, \ldots$

The first part of this Proposition is due to P. Billingsley ([2], Problem 1.1(b), p. 15) (instead of the existence of at least one atom, Billingsley supposes the probability space is discrete $=$ purely atomic but this extra restriction is not essential).

Remark 1.1. In a purely atomic probability space there is no infinite sequence of independent events $\left\{B_{n}, n=1,2, \ldots\right\}$ whose probabilities are bounded away from 0 and 1 , that is, $0<d<P\left(B_{n}\right)<1-d<1$ cannot hold. It is enough to refer to the fact that convergence in probability and a.s. convergence are equivalent if (and only if) $P$ is purely atomic (see Thm 1.5.2 in [1). But the indicator functions of the of events $B_{1}, \bar{B}_{1}, B_{1} B_{2}, B_{1} \bar{B}_{2}, \bar{B}_{1} B_{2}, \bar{B}_{1} \bar{B}_{2}, \ldots$ tend to 0 in probability (the probability of the intersection of $k$ events from $\left\{B_{n}\right\}$ or $\left\{\bar{B}_{n}\right\}$ is less than $\left.(1-d)^{k}\right)$ while the convergence of the same sequence is not a.s. In particular, a discrete probability space cannot contain an infinite sequence of independent events each of probability 1/2 (see Billingsley [2] Problem 1.1(a)).

The range of a purely atomic probability measure can easily be the whole $[0,1]$, e.g. if the probability of the $n$-th atom is $a_{n}=1 / 2^{n+1}$.

Theorem 1.1. If the range $\{P(A): A \in \mathcal{A}\}$ of a probability measure $P$ contains the whole interval $[0,1]$ or at least if the range contains an arbitrary small interval $[0, \varepsilon], \varepsilon>0$, then there are infinitely many independent events in the underlying probability space.

Remark 1.2. Rényi proved (see [4], pp. 80-83) the following. The range of a probability measure is always closed (topologically). If the probability measure is purely atomic with infinitely many atoms, then the range is topologically perfect while in the atomless case the range is always the whole $[0,1]$.

Remark 1.3 . It is very easy to see that if the range is $[0,1]$, then we always have two nontrivial independent events. It is enough to consider purely atomic probability spaces, and we can choose our above-mentioned $\left\{\mathbb{N}_{0}, \mathcal{A}, P\right\}$ space. Choose $A=$ $\{0,1,2, \ldots, n\}$ and $A B=\{n\}$. Then by the independence of $A$ and $B$ we have $P(B \backslash A)=P(A B)(1 / P(A)-1)$. Because the range of $P$ is $[0,1]$, we surely have an event $C$ with probability $P(B \backslash A)$. If $n$ is big enough, e.g. if $P(A)>1 / 2$, then $P(C)<a_{n}$, hence $C$ does not intersect $\{0,1,2, \ldots, n\}$. Finally, set $B=A B \cup C=$ $\{n\} \cup C$.

This argument does not seem to extend for more than two events. We need an entirely different approach in the proof of Theorem 1.1 below.

\section{Proofs}

Proof of Proposition 1.1. If $B_{1}, B_{2}, \ldots$ are independent events and $A$ is an atom, then without loss of generality we can suppose that all of the events $B_{1}, B_{2}, \ldots$ contain $A$. (If this were not the case for some of them, we can replace them by their 
complements, they remain independent.) Denote the probabilities of $A, B_{1}, B_{2}, \ldots$ by $a, p_{1}, p_{2}, \ldots$, resp.

Then for every $n \in \mathbb{N}_{0}$,

$$
\begin{aligned}
a & \leq P\left(B_{1} B_{2} \ldots B_{n}\right)=P\left(B_{1}\right) P\left(B_{2}\right) \ldots P\left(B_{n}\right)=p_{1} p_{2} \ldots p_{n} \\
& <\exp \left\{-\left(1-p_{1}\right)\right\} \cdots \exp \left\{-\left(1-p_{n}\right)\right\}
\end{aligned}
$$

thus $\left(1-p_{1}\right)+\left(1-p_{2}\right)+\cdots \leq \log (1 / a)$, and so this is finite.

Conversely, suppose that this sum is finite. By the Borel-Cantelli lemma we have that at most finitely many $\bar{B}_{n}$ can occur a.s. Thus, with probability 1 , there are only countably many intersections in which every event $B_{n}$ or its complement occurs. These disjoint intersections can be considered as atoms of the $\sigma$-field generated by $B_{1}, B_{2}, \ldots$ (for more details see below): they cover the whole space.

Remark 2.1. The argument above shows that in a probability space with at least one atom there cannot be more than countably many independent events. As we have seen without the loss of generality we can assume that all supposedly independent events contain the same atom. Thus the sum of the probabilities of their complements is uniformly bounded; hence for every positive $\varepsilon$ there are only finitely many events with probability at most $1-\varepsilon$. This implies our claim if $\varepsilon>0$ tends to 0 on a countable set.

From the proof of Proposition 1.1 we can see that there is a simple relation between the probabilities $p_{1}, p_{2}, \ldots$ of our independent events and the probabilities of the atoms generated by $B_{1}, B_{2}, \ldots$ Denote the probabilities of these atoms by $a_{0}, a_{1}, a_{2}, \ldots$ If we suppose that $\sum_{n=1}^{\infty}\left(1-p_{n}\right)<\infty$, then $c:=\prod_{n=0}^{\infty} p_{n}<\infty$. Now let $S$ be a finite subset of $\{1,2, \ldots\}$. Then the atoms of the $\sigma$-field generated by $B_{1}, B_{2}, \ldots$ are $A_{S}=\prod_{i \notin S} B_{i} \prod_{i \in S} \bar{B}_{i}$. These atoms correspond in a unique way to the nonnegative integers $n(S)=\sum_{i \in S} 2^{i}$. Now, it is clear that the probabilities of the atoms are the following:

$$
a_{n}=a_{n(S)}=P\left(A_{S}\right)=c \prod_{i \in S} \frac{1-p_{i}}{p_{i}} .
$$

On the other hand,

$$
B_{i}=\bigcup_{S: i \notin S} A_{S}
$$

Example 2.1. Choose $p_{i}$ such that $\frac{1-p_{i}}{p_{i}}=1 / 2^{2^{i}}$, that is, $p_{i}=1 /\left(1+2^{-2^{i}}\right)$. Then one can easily compute that $\prod_{i \in S} \frac{1-p_{i}}{p_{i}}=1 / 2^{n}$ where $n=n(S)$, and $c=1 / 2$. Hence we have a very simple purely atomic probability space where the probability of the $n$-th atom is $a_{n}=1 / 2^{n+1}$. The atom $n=n(S)$ is contained in the event $B_{i}$ iff $2^{i}$ is not there in the binary expansion of $n$, that is, $B_{i}=\{m: m \equiv$ $\left.1,2, \ldots, 2^{i}\left(\bmod 2^{i+1}\right)\right\}$ are mutually independent.

Proof of Theorem 1.1. Using the arguments above we can we easily check if a purely discrete probability space with given probabilities $a_{0} \geq a_{1} \geq a_{2} \geq \ldots$ of atoms is generated by a sequence of independent events. If the following process never ends, then we obviously have an infinite sequence of independent events that generates our probability space; otherwise we do not have such a sequence of independent events. 
The formula for $a_{n}$ above shows that the maximum probability of atoms $\left(a_{0}\right)$ corresponds to the case when $S$ is empty. Then $a_{0}=c$. Delete $A_{\emptyset}$. Using the notation $b_{i}=\left(1-p_{i}\right) / p_{i}$ the next biggest probability of the remaining atoms is $c b_{1}$. Delete the atom with this probability (if there are more than one atoms with this probability, then delete one of them). The next biggest probability is $c b_{2}$. Delete the corresponding atom. If there is an atom with probability $c b_{1} b_{2}$, then we delete it; otherwise stop and say that our $\sigma$-field is not generated by independent events. The next biggest probability of atoms is $c b_{3}$. Delete the corresponding atom. Delete also the atoms with probabilities $c b_{1} b_{3}, c b_{2} b_{3}, c b_{1} b_{2} b_{3}$. If there are no atoms with any of these probabilities, we stop. The next biggest probability of atoms is $c b_{4}$, etc.

The algorithm above is a necessary and sufficient criterion for a purely atomic $\sigma$-field to be generated by an infinite sequence of independent events. Although it is hard to apply, by using this idea we can get nice sufficient conditions - one of them is our Theorem 1.1.

If the range of a probability measure is the whole interval $[0,1]$ or at least $[0, \varepsilon]$, then the above algorithm shows that there always exists infinitely many independent events in the space. More precisely, if the probabilities $p_{1} \geq p_{2} \geq \ldots$ are given in a such a way that $c:=\prod_{i=1}^{\infty} p_{i} \geq 1-\varepsilon$, then one can always find independent events with probabilities $p_{1}, p_{2}, \ldots$.

We just need the following simple modification of the algorithm above. Instead of looking for an atom $A_{S}, S \neq \emptyset$, with probability $c \prod_{i \in S} b_{i}$, it suffices to find an event in $\mathcal{A}$, disjoint from all previously chosen events, with the prescribed probability. This is always possible since the probability in question is small enough:

$$
\sum_{S \neq \emptyset} P\left(A_{S}\right)=c\left(\prod_{i=1}^{\infty}\left(1+b_{i}\right)-1\right)=1-c \leq \varepsilon .
$$

Thus in each step the range of the probability measure restricted to the complement of all previously chosen events still contains a sufficiently large interval. Finally, let $A_{\emptyset}=\prod_{S \neq \emptyset} \bar{A}_{S}$ and $B_{i}=\bigcup_{S: i \notin S} A_{S}$.

\section{ACKNOWLEDGMENT}

The authors are grateful to Larry Shepp and Truc Nguyen for some valuable advice.

\section{REFERENCES}

[1] Borkar, V. S. (1995). Probability Theory, Springer-Verlag, New York. MR 98e:60001

[2] Billingsley, P. (1995). Probability and Measure, 3rd ed. J. Wiley \& Sons, New York. MR 95k:60001

[3] Chen, Zh., Rubin, H., Vitale, R. A. (1997). Independence and determination of probabilities, Proc. Amer. Math. Soc., 125 no. 12, 3721-3723. MR 98b:60007

[4] Rényi, A. (1970). Probability Theory, North-Holland, New York. MR 47:4296

Department of Mathematics and Statistics, Bowling Green State University, Bowling Green, Ohio 43403-0221 and Rényi Institute of Mathematics, Hungarian Academy of Sciences, P.O.B. 127, 1364 Budapest, Hungary

E-mail address: gabors@bgnet.bgsu.edu

Department of Probability and Statistics, Eötvös L. University, Kecskeméti u. 10,1093 Budapest, Hungary

E-mail address: moritamas@ludens.elte.hu 